\title{
Continual model and dynamic calculation of buildings under seismic impacts
}

\author{
M. K. Usarov*, G. T. Ayubov, and D. M. Usarov \\ Institute of Mechanics and Seismic Stability of Structures of the Academy of Sciences of the \\ Republic of Uzbekistan, 31 Durmon yuli str., Tashkent 100125, Uzbekistan
}

\begin{abstract}
Continuum plate model in the form of a cantilever anisotropic plate developed in the framework of the bimoment theory of plates describing seismic oscillations of buildings is proposed in this paper as a dynamic model of a building. Formulas for the reduced moduli of elasticity, shear and density of the plate model of a building are given. Longitudinal oscillations of a building are studied using the continuum plate and box-like models of the building with Finite Element Model. Numerical results are obtained in the form of graphs, followed by their analysis.
\end{abstract}

\section{Introduction}

Studies in the theory of structures showed that in strength calculation, the study of dynamic behavior and assessment of the stress-strain state of various structures, taking into account physical and geometrical nonlinearity, viscoelastic and anisotropic properties of the material, as well as inhomogeneous structural features under the influence of external impacts are important.

The effect of earthquakes of various intensities and frequency on the seismic resistance of a wooden building was considered in [1]. Evaluation of seismic resistance was made on the basis of experimental and design-theoretical calculations. Based on the obtained experimental data, a design-theoretical assessment of the frame building was performed on simple and complex models under the effect of earthquakes of various intensities and frequency content.

Structurally-nonlinear problems with one-way connections are often encountered in calculation of various types of structures and buildings. Problems considering the friction on the contact and under dynamic action of load present certain difficulties. The study in [2] is devoted to the construction of computational models and methods for solving problems with non-ideal one-way connections under dynamic loading.

In [3-5], various dynamic problems were considered, devoted to the estimation and prediction of dynamic behavior of various structures, taking into account physical and geometrical nonlinearity, inhomogeneous structural features under multicomponent kinematic effect.

\footnotetext{
*Corresponding author: umakhamatali@mail.ru
} 
In [6] the solution to the problem of optimizing the projects of industrial buildings designed in seismically hazardous areas was considered. Economic efficiency was taken as an optimality criterion, depending on certain variable parameters adopted at the design stage.

In [7], the problem of evaluating the impact of wall panel fractures when estimating large-panel structures strength was solved. The forces exceeding the permissible values in structural elements were calculated

The model of a box-like structure of a building is improved in [8,9] taking into account forces and moments in the contact zones of beam and plate elements interaction. The equations of motion of the box-like elements, the boundary conditions in the box base and the contact conditions between the box elements are given; the graphs of plates and beams displacements are constructed. The problem of forced oscillations of a building of spatial box type is considered in the paper; it composes of rectangular panels and interacting beams under dynamic effect set by base displacement according to a sinusoidal law. The finite difference method was used in the problem solution. Numerical results of stresses, displacements in the hazard areas of the box-like building are obtained.

The studies in $[10,11]$ are devoted to the development of the methods for dynamic spatial calculation of a structure based on the finite difference method in the framework of the bimoment theory, which takes into account the spatial stress-strain state.

Solutions to the problem of transverse and longitudinal vibrations of buildings and structures were obtained using a plate model developed in the framework of the bimoment theory of plates $[12,13]$.

There are numerous articles and monographs devoted to the development of the theory of seismic resistance of a building. Various methods for calculating buildings and structures for seismic actions have been developed, taking into account important factors, such as seismic loading, soil conditions of terrain and structural features of buildings [10]. Note that the analysis of the consequences of strong earthquakes has shown the shortcomings of existing methods of calculating buildings and structures for seismic resistance. One of the most common design schemes of the building is a multi-mass elastic cantilever rod. Oscillations of a spatial construction are reduced to the consideration of oscillations of a plane system consisting of several concentrated masses connected by certain rigidities. Many researchers, criticizing the cantilever design of buildings, recognize the need to move to improved calculation schemes that are more adequate to real structures.

\section{Statement of the problem}

To describe the motion of the plate model of a building, introduce a Cartesian coordinate system with variables $x_{1}, x_{2}$ and $z$. The origin is located in the lower left corner of the median surface. We direct the axes $O X_{1}$ and $O X_{2}$ along the length and height, and the axis $O Z$ - along the thickness (the width of the building) of a plate model. Assume that seismic motion of soil occurs in the direction of the axis $O X$ (the length of the building).

In [10], formulas for the reduced density and moduli of elasticity and shear of a plate model of the building are obtained. Mechanical and physical characteristics of the building are defined under the assumption that the building consists of numerous boxes (rooms) with volumes determined by the formula:

$$
V_{\text {box }}=d_{1} d_{2} d_{3}, V_{0}=2\left(\frac{d_{1} d_{2} H_{3}}{2}+\frac{d_{1} d_{3} H_{2}}{2}+\frac{d_{2} d_{3} H_{1}}{2}\right),
$$

where $d_{1}, d_{2}$ - are the dimensions of the building box in plan, $d_{3}$ - the height of the box, 
$V_{0}$ - is the sum of the volumes of bearing and room partitions and floors, $\mathrm{H}_{1}, \mathrm{H}_{2}$ - is the thickness of bearing and room partitions, $\mathrm{H}_{3}$ - is the thickness of the floor.

Remaining reduced elastic characteristics and densities of the building are determined by the formulas:

$$
\begin{gathered}
E_{1}^{(\mathrm{bd})}=\xi_{11} E_{0}, E_{2}^{(\mathrm{bd})}=\xi_{22} E_{0}, E_{3}^{(\mathrm{bd})}=\xi_{33} E_{0}, \\
G_{12}^{(\mathrm{bd})}=\xi_{12} E_{0}, G_{13}^{(\mathrm{bd})}=\xi_{13} E_{0}, G_{23}^{(\mathrm{bd})}=\xi_{23} E_{0}, \rho_{\mathrm{bd}}=\rho_{0} \xi_{0} .
\end{gathered}
$$

where $E_{0}$ - is the modulus of elasticity of building material

The values of the coefficients $\xi_{11}, \xi_{22}, \xi_{33}, \xi_{12}, \xi_{13}, \xi_{23}, \zeta_{0}$ for each building cell (room) are defined as functions of two spatial variables, $E_{0}, G_{0}$ - are the moduli of elasticity and shear of the strongest bearing panel of the building. Reduced modulus of elasticity of a building is determined by the formula

$$
\xi_{11}=\frac{S_{11}}{S_{01}}, \quad \xi_{22}=\frac{S_{22}}{S_{02}}, \quad \xi_{33}=\frac{S_{33}}{S_{03}} E_{0}, \quad \xi_{12}=\frac{S_{11}}{S_{01}}, \quad \xi_{13}=\frac{h_{f p l}}{b_{1}} \lambda^{*}, \quad \xi_{23}=\frac{h_{2}}{b}, \quad \zeta_{0}=\frac{V_{0}}{V_{\text {box }}},
$$

where $S_{01}, S_{02}, S_{03}$ - are the cross-sectional areas of the building in three coordinate planes of one floor of the building; $S_{11}, S_{22}, S_{33}$ - are the total areas of cross sections of the plates in the coordinate planes, forming one floor of the building; $\lambda^{*}-$ is a coefficient characterizing the voids in the cross-section of the floor plate[10].

Note that the above volumes and areas are determined, depending on the dimensions of plates, rooms and the building itself, as follows:

$$
\begin{array}{r}
S_{01}=b_{1} H, S_{02}=a H, S_{03}=a b_{1}, S_{11}=2 b_{1} h_{1}+b_{1} h_{2}+H h_{\mathrm{fpl}}, \\
S_{22}=2 a h_{1}+a h_{2}+2 H h_{1}+(k-2) H h_{2}, S_{33}=2 b_{1} h_{1}+a h_{2}+(k-2) b_{1} h_{2} .
\end{array}
$$

The values of coefficients $\xi_{11}, \xi_{22}, \xi_{33}, \xi_{12}, \xi_{13}, \xi_{23}$ are determined for each cell (room) of the building. In general, these coefficients are variables and are the functions of two spatial coordinates, which should be determined for the building in question from multiple numerical theoretical experiments and existing experimental data.

Longitudinal, tangential forces and bimoments are determined with respect to the following nine unknown kinematic functions:

$$
\begin{gathered}
\bar{u}_{k}=\frac{u_{k}^{(+)}+u_{k}^{(-)}}{2}, \bar{\psi}_{k}=\frac{1}{2 h} \int_{-h}^{h} u_{k} d z, \bar{\beta}_{k}=\frac{1}{2 h^{3}} \int_{-h}^{h} u_{k} z^{2} d z,(k=1,2), \\
\bar{W}=\frac{u_{3}^{(+)}-u_{3}^{(-)}}{2}, \bar{r}=\frac{1}{2 h^{2}} \int_{-h}^{h} u_{3} z d z, \bar{\gamma}=\frac{1}{2 h^{4}} \int_{-h}^{h} u_{3} z^{3} d z .
\end{gathered}
$$

Where $u_{1}\left(x_{1}, x_{2}, z, t\right), u_{2}\left(x_{1}, x_{2}, z, t\right), u_{3}\left(x_{1}, x_{2}, z, t\right)-$ is the relative displacement of the building points caused by building deformation; $u_{i}^{(-)}, \quad u_{i}^{(+)},(i=1,3)$ - are the displacements of points on the faces of the plate $z=-h$ and $z=+h$. Function $r$ expresses an average value of normal displacements of the plate model of the building; $\bar{W}$ - is a half-difference of normal displacements of two outer layers of the plate model; functions $\bar{u}_{1}$ and $\bar{u}_{2}$ - are the half-sums of longitudinal displacements, representing the displacements of the outer layers of the plate model of the building along horizontal and vertical directions. 
Let's introduce the elastic constants of Hooke's law $E_{11}, E_{12}, \ldots, E_{33}$, which determine the components of the stress tensor via strain tensor's components for threedimensional cases [13].

The equations of longitudinal oscillations of a thick plate [11-13], built with internal forces and bimoments within the framework of the spatial theory of elasticity, are taken as the equation of motion of a building under seismic action directed along the longitudinal direction, and are written in the following form

$$
\begin{gathered}
\frac{\partial N_{11}}{\partial x_{1}}+\frac{\partial N_{12}}{\partial x_{2}}-\rho H \ddot{\bar{\psi}}_{1}=\rho H \ddot{U}_{0}, \frac{\partial N_{21}}{\partial x_{1}}+\frac{\partial N_{22}}{\partial x_{2}}=\rho H \ddot{\bar{\psi}}_{2}, \\
\frac{\partial T_{11}}{\partial x_{1}}+\frac{\partial T_{12}}{\partial x_{2}}-4 \bar{p}_{13}-\rho H \ddot{\bar{\beta}}_{1}=\frac{1}{3} \rho H \ddot{U}_{0}, \frac{\partial T_{12}}{\partial x_{1}}+\frac{\partial T_{22}}{\partial x_{2}}-4 \bar{p}_{23}=\rho H \ddot{\bar{\beta}}_{2}, \\
\frac{\partial \bar{p}_{13}}{\partial x_{1}}+\frac{\partial \bar{p}_{23}}{\partial x_{2}}-\frac{2 \bar{p}_{33}}{H}=\rho \ddot{\bar{r}}, \frac{\partial \bar{\tau}_{13}}{\partial x_{1}}+\frac{\partial \bar{\tau}_{23}}{\partial x_{2}}-\frac{6 \bar{\tau}_{33}}{H}=\rho \ddot{\bar{\gamma}}
\end{gathered}
$$

The equations of motion for determining the displacements of external longitudinal walls, obtained by meeting the boundary conditions on the faces of the plate $z=-h$ and $z=+h$ using the method of displacements expansion into the Maclaurin infinite power series, are constructed in $[12,13]$ in the form:

$$
\begin{aligned}
& \bar{W}=\frac{1}{2}(21 \bar{\gamma}-7 \bar{r})-\frac{1}{30} H\left(\frac{E_{31}}{E_{33}} \frac{\partial \bar{u}_{1}}{\partial x_{1}}+\frac{E_{32}}{E_{33}} \frac{\partial \bar{u}_{2}}{\partial x_{2}}\right) \\
& \bar{u}_{k}=\frac{1}{4}\left(21 \bar{\beta}_{k}-3 \bar{\psi}_{k}\right)-\frac{1}{20} H \frac{\partial \bar{W}}{\partial x_{k}}+\frac{1}{20} \frac{H \bar{q}_{k}}{G_{k 3}} \quad(k=1,2) .
\end{aligned}
$$

Expressions of longitudinal and tangential forces are written in the form:

$$
\begin{gathered}
N_{11}=E_{11} H \frac{\partial \bar{\psi}_{1}}{\partial x_{1}}+E_{12} H \frac{\partial \bar{\psi}_{2}}{\partial x_{2}}+2 E_{13} \bar{W}, \quad N_{22}=E_{12} H \frac{\partial \bar{\psi}_{1}}{\partial x_{1}}+E_{22} H \frac{\partial \bar{\psi}_{2}}{\partial x_{2}}+2 E_{23} \bar{W}, \\
N_{12}=N_{21}=G_{12}\left(H \frac{\partial \bar{\psi}_{1}}{\partial x_{2}}+H \frac{\partial \bar{\psi}_{2}}{\partial x_{1}}\right) .
\end{gathered}
$$

Expressions of longitudinal and tangential bimoments have the form:

$$
\begin{gathered}
T_{11}=H\left(E_{11} \frac{\partial \bar{\beta}_{1}}{\partial x_{1}}+E_{12} \frac{\partial \bar{\beta}_{2}}{\partial x_{2}}+E_{13} \frac{2 \bar{W}-4 \bar{r}}{H}\right), \quad T_{22}=H\left(E_{12} \frac{\partial \bar{\beta}_{1}}{\partial x_{1}}+E_{22} \frac{\partial \bar{\beta}_{2}}{\partial x_{2}}+E_{23} \frac{2 \bar{W}-4 \bar{r}}{H}\right), \\
T_{12}=T_{21}=H G_{12}\left(\frac{\partial \bar{\beta}_{1}}{\partial x_{2}}+\frac{\partial \bar{\beta}_{2}}{\partial x_{1}}\right)
\end{gathered}
$$

Bimomentsare expressed in the form

$$
\bar{\sigma}_{11}=E_{11}^{*} \frac{\partial \bar{u}_{1}}{\partial x_{1}}+E_{12}^{*} \frac{\partial \bar{u}_{2}}{\partial x_{2}}, \quad \bar{\sigma}_{12}=G_{12}\left(\frac{\partial \bar{u}_{1}}{\partial x_{2}}+\frac{\partial \bar{u}_{2}}{\partial x_{1}}\right), \quad \bar{\sigma}_{22}=E_{12}^{*} \frac{\partial \bar{u}_{1}}{\partial x_{1}}+E_{22}^{*} \frac{\partial \bar{u}_{2}}{\partial x_{2}} .
$$

Intensities of transverse and normal bimoments $\bar{p}_{13}, \bar{p}_{23}, \bar{\tau}_{13}, \bar{\tau}_{23}$ и $\bar{p}_{33}, \bar{\tau}_{33}$ have the form:

$$
\begin{gathered}
\bar{p}_{k 3}=G_{k 3}\left(\frac{\partial \bar{r}}{\partial x_{k}}+\frac{2\left(\bar{u}_{k}-\bar{\psi}_{k}\right)}{H}\right), \bar{\tau}_{k 3}=G_{k 3}\left(\frac{\partial \bar{\gamma}}{\partial x_{k}}+\frac{2\left(\bar{u}_{k}-3 \bar{\beta}_{k}\right)}{H}\right),(k=1,2) \\
\bar{p}_{33}=E_{31} \frac{\partial \bar{\psi}_{1}}{\partial x_{1}}+E_{32} \frac{\partial \bar{\psi}_{2}}{\partial x_{2}}+E_{33} \frac{2 \bar{W}}{H}, \bar{\tau}_{33}=E_{31} \frac{\partial \bar{\beta}_{1}}{\partial x_{1}}+E_{32} \frac{\partial \bar{\beta}_{2}}{\partial x_{2}}+E_{33} \frac{2 \bar{W}-4 \bar{r}}{H} .
\end{gathered}
$$

Intensities of bimoments $\bar{\sigma}_{11}^{*}, \quad \bar{\sigma}_{22}^{*}, \tilde{\sigma}_{11}^{*}, \tilde{\sigma}_{22}^{*}$ are expressed as: 

$E_{23} \frac{\bar{R}}{H^{\prime}}$

$$
\bar{\sigma}_{11}^{*}=-E_{11} H \frac{\partial^{2} \bar{W}}{\partial x_{1}^{2}}-E_{12} H \frac{\partial^{2} \bar{W}}{\partial x_{2}^{2}}+E_{13} \frac{\bar{R}}{H^{\prime}} \quad \bar{\sigma}_{22}^{*}=-E_{12} H \frac{\partial^{2} \bar{W}}{\partial x_{1}^{2}}-E_{22} H \frac{\partial^{2} \bar{W}}{\partial x_{2}^{2}}+
$$

Where $\bar{R}=420(\bar{W}+6 \bar{r}-15 \bar{\gamma})$.

The system of differential equations of motion (6) - (10) constitutes a joint system of seven equations with respect to nine unknown functions $\bar{\psi}_{1}, \bar{\psi}_{2}, \bar{\beta}_{1}, \bar{\beta}_{2}, \bar{u}_{1}, \bar{u}_{2}$, $\bar{r}, \bar{\gamma}, \bar{W}$.

Let us write down the boundary conditions for the problem of bending and shear vibrations of buildings. Suppose that the foundation points move according to a given law $u_{0}(t)$, and the lower part of the building moves horizontally with the foundation. From kinematic considerations it follows that the displacements will be written in the form:

$$
u\left(x_{1}, 0, z, t\right)=u_{0}(t), \vartheta\left(x_{1}, 0, z, t\right)=0, w\left(x_{1}, 0, z, t\right)=0 .
$$

From kinematic conditions it follows that in the foundation of the building the following boundary conditions must be fulfilled:

$$
\bar{\psi}_{1}=0, \quad \bar{\psi}_{2}=0, \quad \bar{\beta}_{1}=0, \quad \bar{\beta}_{2}=0, \bar{r}=0, \quad \bar{\gamma}=0, \quad \bar{u}_{1}=0, \quad \bar{u}_{2}=0, \bar{W}=0
$$

On the free side faces of the building the conditions of zero force factors are fulfilled:

$$
N_{11}=0, N_{12}=0, T_{11}=0, T_{12}=0, \quad \bar{\sigma}_{11}=0, \quad \bar{\sigma}_{12}=0, \quad \bar{p}_{13}=0, \quad \bar{\tau}_{13}=0, \quad \bar{\sigma}_{11}^{*}=0 .
$$

On the free top faces of the building the conditions of zero force factors are fulfilled:

$$
N_{12}=0, N_{22}=0, \quad T_{12}=0, \quad T_{22}=0, \quad \bar{\sigma}_{12}=0, \quad \bar{\sigma}_{22}=0, \quad \bar{p}_{23}=0, \quad \bar{\tau}_{23}=0, \quad \bar{\sigma}_{22}^{*}=0 .
$$

The problem is solved by the Finite Differences Method. To approximate the first derivatives, the central difference schemes are taken.

\section{Method of the solution}

The problem is solved by the finite difference method. To approximate the first derivatives, the central difference schemes is taken.

To substantiate the expressions obtained with continuous model of calculation, consider a specific problem with the use of necessary physical and mechanical characteristics of a box-like model. Calculation of the box-like models has been carried out by the FEM, the main point of which is the replacement of a real system (a multi-story box-like building) with a discrete model of rectangular plane elements (walls and floors) connected at the nodal points and satisfying the equilibrium conditions of the converging system of forces at each node.

Mechanical characteristics and geometry of the rooms panels are accepted as follows: bending bearing panels have a modulus of elasticity $E=20000 \mathrm{MPa}$; density $\rho=2700 \frac{\mathrm{kg}}{\mathrm{m}^{3}}$; Poisson's ratio $v=0.3$. For the panel working on shear, the following mechanical characteristics are adopted: modulus of elasticity $E=7500 \mathrm{MPa}$, density $\rho=1200 \frac{\mathrm{kg}}{\mathrm{m}^{3}}$; Poisson's ratio $v=0.3$.

Results of calculations of forced oscillations of a building within the framework of a thick plate model with the following dimensions of the floor slab of the building are:

$h_{1}=0.25 \mathrm{~m}, h_{2}=0.25 \mathrm{~m}, h_{\mathrm{fpl}}=0.2 \mathrm{~m}, a_{1}=7.5 \mathrm{~m}, b_{1}=3 \mathrm{~m}, a=30 \mathrm{~m}, H=11 \mathrm{~m}$. 
In all cases the effect is given by the acceleration of the foundation at amplitude $a_{0}=0.1 \mathrm{~g}$, i.e. at unit amplitude, which corresponds to the intensity of the 7-point earthquake

$$
\ddot{U}_{0}=a_{0} \cos \left(2 \pi \omega_{0} t\right) .
$$

The effect frequency is chosen equal to $\omega_{0}=9.5 \mathrm{~Hz}$. Such a high-frequency effect has a period of T $=0.1 \mathrm{~s}$. The Gazli earthquake (1976) accelerogram had such predominant period. So, it can be said that the considered effect is in a certain sense analogous in predominant period to the Gazli earthquake (1976). The scheme of the building in question is shown in Figure. 1.

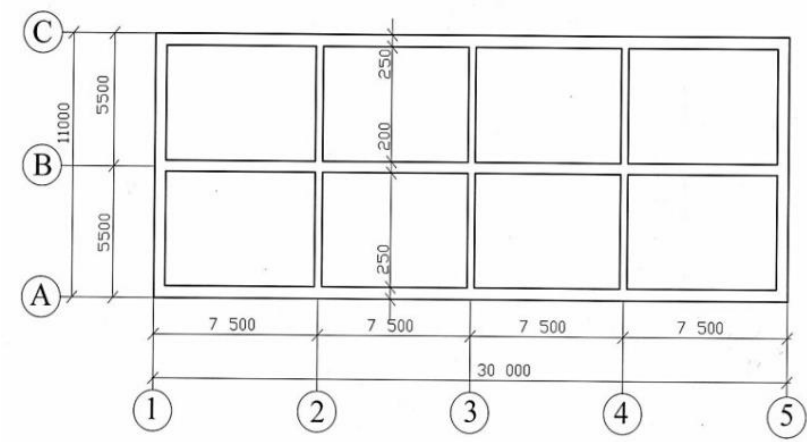

Fig. 1. Scheme of the box-like model of a building.

\section{Solution to the test problem}

Results obtained with FEM and FDM for the box-like and the continuum plate model of a building are shown in the form of graphs in Figures. 2.

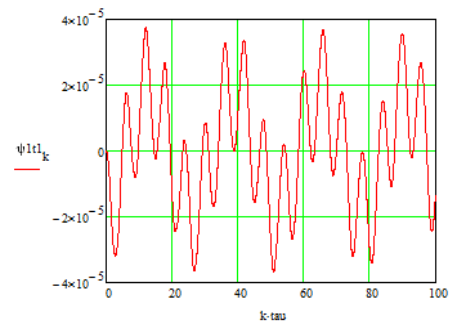

a)

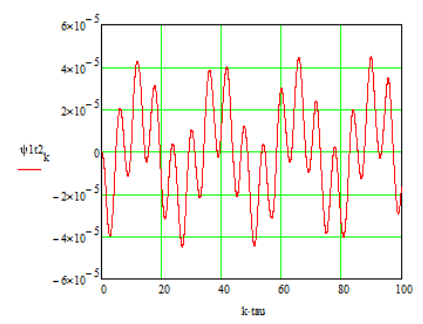

b)

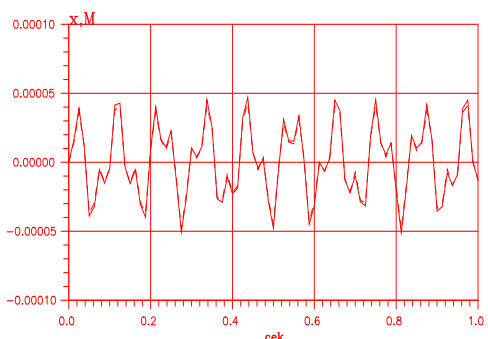

c)

Fig. 2. Displacements of a two-story building, obtained at characteristic points, in $m$.

$a$ )- are the characteristic points at the middle of the floor, $b$ )are the characteristic points at the edge of the floor, $c$ ) is the graph obtained with FEM. 
Figure 2 shows oscillations of characteristic points located at the level of building floors. Solid line characterizes the points at the edge of the floor, and the dashed line - the points at the center of the floor. Under given effects the oscillations of points on the right and left edges of the floor merge.

Figures $2 \mathrm{a}$ and $2 \mathrm{~b}$ show the displacements of the middle and edge points of the slab on the basis of the plate continuum model of a two-story building and their values are $\psi_{1}=0.0000374 \mathrm{~m}$ and $\psi_{1}=0.0000449 \mathrm{~m}$, respectively. Fig. 2c shows the values of displacements at the middle and edge points of the slab on the basis of the box-like model with FEM of a two-story building and their values are $R_{\text {mid }}=0.0000430 \mathrm{~m}$ and $R_{\text {edg }}=0.0000500 \mathrm{~m}$, respectively.

Table 1 shows the maximum values of the generalized displacement and acceleration $\psi_{1}$ and $\ddot{\psi}_{1}$, obtained under forced longitudinal vibrations of a two-story building at extreme points in the upper levels of the first and second floors.

Table 1. Maximum values of displacements and accelerations of a two-story building under longitudinal vibrations

\begin{tabular}{|c|c|c|}
\hline $\begin{array}{c}\text { Number of floors in the } \\
\text { building }\end{array}$ & $\psi_{1}, 10^{-4} \mathrm{~m}$ & $\ddot{\psi}_{1}, \mathrm{~m} / \mathrm{s}^{2}$ \\
\hline 1 & 0.2878 & 2.798 \\
\hline 2 & 0.4491 & 3.754 \\
\hline
\end{tabular}

Calculations showed that under longitudinal vibrations of multi-story buildings, the maximum values of normal stresses $\sigma_{11}$ and $\sigma_{22}$ are reached at the extreme points of the lower part of the external load-bearing wall with coordinates $x_{1}=a, y_{1}=0$.

Figures 3 and 4 show graphs of changes in dimensionless time $\tau$ of normal stresses $\sigma_{11}$ and $\sigma_{22}$ in the external load-bearing wall of a two-story building.

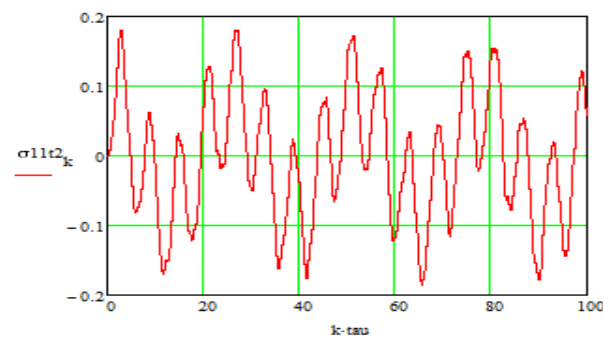

Fig. 3. Graph of stress $\sigma_{11}$ over time of a twostory building under longitudinal vibrations.

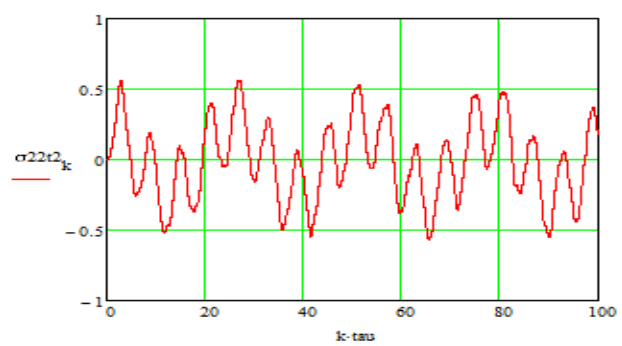

Figure 4. Graph of stress $\sigma_{22}$ over time of a twostory building under longitudinal vibrations.

Figure 3 shows a graph of stress $\sigma_{11}$ variation over dimensionless time $\tau$, according to which the maximum stress value is set equal to $\sigma_{11}=0.192 \mathrm{MPa}$.

Figure 4 shows a graph of the change in dimensionless time $\tau$, of the normal stress $\sigma_{22}$ in the external load-bearing wall of the building at the extreme lower points. The maximum value of normal stress is equal to $\sigma_{22}=0.611 \mathrm{MPa}$. 
When comparing results based on plotted graphs for the box-like model with FEM and the plate continuum model, it can be seen that the values of displacements do not differ significantly.

\section{Conclusions}

Comparison of results based on the plotted graphs shows that the values of displacements based on the box-like model with FEM are 10-13\% less than the results based on the plate continuum model.

In conclusion, it should be noted that on the basis of the bimoment theory of thick plates a dynamic plate-like model of the building has been developed, reflecting its spatial strains. Using the geometry of the building and its rooms, the reduced density, moduli of elasticity and shear of the plate model have been determined. The plate model of the building due to the choice of coefficients $\xi_{22}, \xi_{33}, \xi_{12}, \xi_{13}, \xi_{23}$, adequately reflects the laws of displacements of the points of the building. So, the plate model is acceptable for determining the displacement fields of a tall building during an earthquake and the dynamic characteristics of a building.

\section{References}

1. Belash T A, Ivanova Zh V 2019 Wooden buildings of frame type with effective designs of node connections for seismically active areas Magazine of Civil Engineering (St. Petersburg: Peter the Great St. Petersburg Polytechnic University) 8 (92) 84-95 DOI: 10.18720 / mce.92.7

2. Lukashevich A 2019 Modeling the contact interaction of structures with the base under dynamic loading Magazine of Civil Engineering (St. Petersburg: Peter the Great St. Petersburg Polytechnic University) 5 (89) 167-178 DOI: 10.18720 / mce.89.14

3. Mirsaidov M M and Sultanov T Z 2014 Assessment of stress-strain state of earth dams with allowance for non-linear strain of material and large strains Magazine of Civil Engineering 49 73-82DOI: $10.5862 /$ mce.49.8

4. Ishmatov A N and Mirsaidov M 1991 Nonlinear vibrations of an axisymmetric body acted upon by pulse loads Soviet Applied Mechanics 27 388-394 DOI: $10.1007 / \mathrm{BF} 00896519$

5. Radin V.P., Trifonov O.V., Chirkov V.P. A model of a multi-story frame building for calculations of intense seismic effects // Earthquake-resistant construction. Safety of facilities. 2001. No. 1. C. 23-26

6. Vatin N I, Ivanov A Y, Rutman Y L, Chernogorskiy S A and Shvetsov K V 2017 Earthquake engineering optimization of structures by economic criterion Magazine of Civil Engineering 76 67-83 DOI:10.18720/ mce.76.7

7. Vatin N I, Kuznetsov V D and Nedviga E S 2011 Installation errors in calculating large-panel buildings Magazine of Civil Engineering 24 35-40 DOI:10.5862/mce.24.3

8. Usarov M, Mamatisaev G, Yarashov J and Toshmatov E 2020 Non-stationary oscillations of a box-like structure of a building Journal of Physics: Conference Series 1425012003

9. Usarov M, Mamatisaev G, Toshmatov E and Yarashov J 2020 Forced vibrations of a box-like structure of a multi-storey building under dynamic effect Journal of Physics: Conference Series 1425012004

10. Yarashov J, Usarov M and Ayubov G 2019 Study of longitudinal oscillations of a five-storey building on the basis of plate continuum model E3S Web of Conferences 
97 04065 DOI:org/10.1051/e3sconf/20199704065

11. Toshmatov E, Usarov M, Ayubov G and Usarov D 2019 Dynamic methods of spatial calculation of structures based on a plate model E3S Web of Conferences 9704072 DOI:org/10.1051/e3sconf/20199704072

12. Usarov D, Turajonov K and Khamidov S 2020 Simulation of free vibrations of a thick plate without simplifying hypotheses Journal of Physics: Conference Series 1425 012115

13. Usarov M K 2015 Buckling of orthotropic plates with bimoments Magazine of Civil Engineering (St. Petersburg: Peter the Great St. Petersburg Polytechnic University) 53(1) 80-90 DOI: $10.5862 /$ mce.53.8 